\title{
Canonical Wnt signalling activates TAZ through PP1A during osteogenic differentiation
}

\author{
MR Byun ${ }^{1}$, J-H Hwang ${ }^{1}$, AR Kim¹, KM Kim¹, ES Hwang ${ }^{2}$, MB Yaffe ${ }^{*, 3}$ and J-H Hong, ${ }^{*, 1}$
}

TAZ, a transcriptional modulator, has a key role in cell proliferation, differentiation and stem cell self-renewal. TAZ activity is regulated by several signalling pathways, including Hippo, GPCR and Wnt signalling, but the regulatory mechanisms of TAZ activation are not yet clearly understood. In this report, we show that TAZ is regulated by canonical Wnt signalling during osteogenic differentiation. Wnt3a increases TAZ expression and an inhibitor of GSK3 $\beta$, a downstream effector of Wnt signalling, induces TAZ. Wnt3a facilitates the dephosphorylation of TAZ, which stabilises TAZ and prevents it from binding 14-3-3 proteins, thus inducing the nuclear localisation of TAZ. Dephosphorylation of TAZ occurs via PP1A, and depletion of PP1A blocks Wnt3ainduced TAZ stabilisation. Wnt3a-induced TAZ activates osteoblastic differentiation and siRNA-induced TAZ depletion decreases Wnt3a-induced osteoblast differentiation. Taken together, these results show that TAZ mediates Wnt3a-stimulated osteogenic differentiation through PP1A, suggesting that the Wnt signal regulates the Hippo pathway.

Cell Death and Differentiation (2014) 21, 854-863; doi:10.1038/cdd.2014.8; published online 7 February 2014

Transcriptional co-activator with PDZ-binding motif (TAZ, also known as WWTR1) is a transcriptional modulator that has a key role in cell proliferation, differentiation and stem cell selfrenewal. TAZ interacts with several transcription factors including Runx2, PPAR $\gamma$, TEADS, TTF-1/Nkx2.1, Tbx5, Pax3, Smad2/3-4 complexes, MyoD and NFAT5. ${ }^{1-11}$ This interaction regulates the transcription of target genes with diverse biological functions. For example, TAZ and its paralogue YAP interact with TEADs and stimulate their target genes, including CTGF and Cyr61, to promote cell proliferation and migration. This stimulation is inhibited by Hippo signalling, which regulates organ size, cell proliferation, differentiation and stem cell self-renewal. ${ }^{12-15}$ Several components are involved in the transduction of the signal. In Drosophila, Hippo and its regulatory protein Salvador stimulate Warts, which inactivates Yorkie. These components are highly evolutionarily conserved and their mammalian orthologues have been characterised. MST1/2 and its regulatory protein WW45 stimulate the downstream kinase Lats1/2, which inhibits nuclear localisation of TAZ/YAP and induces their proteolytic degradation.

In cellular differentiation, TAZ modulates the cellular fate of mesenchymal stem cells (MSCs) via the activation of osteoblast and myoblast differentiation, and the inhibition of adipocytes differentiation. ${ }^{2,10}$ The interaction between TAZ and Runx2 stimulates Runx2-mediated gene transcription. ${ }^{2}$ However, the regulatory signals that modulate TAZ activity in MSC differentiation are not yet well known.
Wnts are a family of secreted glycoproteins that are involved in the regulation of cell proliferation, differentiation, axis formation, organ development and tissue homeostasis. ${ }^{16-18}$ $\beta$-Catenin is a transcriptional regulator in the canonical Wnt pathway, and, in the absence of Wnts, cytoplasmic $\beta$-catenin is phosphorylated by casein kinase I (CKI) and glycogen synthase kinase $3 \beta$ (GSK3 $\beta$ ). This phosphorylation facilitates its ubiquitination and proteasomal degradation. Canonical Wnt ligands bind to frizzled (FZD) receptors and low-density lipoprotein receptor-related protein 5 (LRP5) or LRP6 co-receptors; this binding inhibits the proteasomal degradation of $\beta$-catenin, increases the stability of cytoplasmic $\beta$-catenin and facilitates the translocation of this protein into the nucleus. ${ }^{19}$ Nuclear $\beta$-catenin interacts with T-cell factor/ lymphoid-enhancing factor (TCF/LEF) transcription factors and activates Wnt target genes. Wnt signalling positively regulates bone development in vivo. A gain of function mutation in the Wnt receptor LRP5 leads to high bone mass in humans. ${ }^{20-22}$ Genetic deletion of LRP5 causes a decrease in bone mass in mice. ${ }^{23}$ Transgenic mice expressing Wnt10b under the control of an osteoblast-specific promoter show increased bone mass. ${ }^{24}$ In contrast, Wnt10b-deleted mice show decreased bone mass. ${ }^{25}$ Inactivation of $\beta$-catenin in osteoblasts led to decreased bone mass caused by increased bone resorption. ${ }^{26-28}$

Recently, TAZ was identified as a downstream effector of Wnt signalling, and the signal stabilises TAZ by inhibiting its proteolytic degradation. ${ }^{29}$ However, the detailed mechanism

\footnotetext{
${ }^{1}$ Division of Life Sciences, Korea University, Seoul, Korea; ${ }^{2}$ College of Pharmacy and Global Top5 Research Program, Ewha Womans University, Seoul, Korea and ${ }^{3}$ Center for Cancer Research, Department of Biology, Massachusetts Institute of Technology, Cambridge, MA, USA

${ }^{*}$ Corresponding authors: MB Yaffe, David H. Koch Institute for Integrative Cancer Research, Massachusetts Institute of Technology, Cambridge, MA 02139, USA. Tel: +1 617452 2442; Fax: +1 617452 4978; E-mail: myaffe@ @it.edu

or J-H Hong, Division of Life Sciences, Korea University, Seoul 136-701, Korea. Tel: +82 23290 3427; Fax: +82 2927 9028; E-mail: jh_hong@korea.ac.kr Keywords: TAZ; Wnt; Hippo pathway; PP1A; osteoblast

Abbreviations: TAZ, transcriptional co-activator with PDZ-binding motif; GSK3 $\beta$, glycogen synthase kinase 3 $\beta$; FZD, frizzled receptor; LRP5, low-density lipoproteinreceptor-related protein 5; LRP6, low-density lipoprotein-receptor-related protein 6; TCF, T-cell factor; LEF, lymphoid-enhancing factor; shRNA, small hairpin RNA; siRNA, small interfering RNA; LiCl, lithium chloride; OSX, osterix; OC, osteocalcin; DMSO, dimethyl sulphoxide; OA, okadaic acid

Received 12.6.13; revised 13.12.13; accepted 02.1.14; Edited by M Piacentini; published online 07.2.14
} 
of TAZ activation in Wnt-stimulated osteoblastic differentiation has not been investigated. Here, we examine the functional connection between a Hippo effector, TAZ and Wnt signalling in osteoblast differentiation. We observed that Wnt3a stabilised TAZ and stimulated osteogenic differentiation through PP1A.

\section{Results}

Wnt3a induces TAZ expression and increases its nuclear localisation. Wnt signalling has an important role in bone development, and TAZ stimulates osteoblast differentiation. To study the functional connection between TAZ activation and Wnt signalling, the expression and cellular localisation of TAZ were observed in Wnt3a-treated C3H10T1/2 cells. As shown in Figure 1a, TAZ expression was induced $12 \mathrm{~h}$ after exposure to Wnt3a. As a control, Wnt3a-induced $\beta$-catenin was analysed, and its induction was observed within $3 \mathrm{~h}$ after Wnt3a treatment. To study whether TAZ induction is derived from transcriptional activation of TAZ, the expression of TAZ mRNA was analysed by quantitative RT-PCR (qRT-PCR). Figure $1 b$ shows that TAZ mRNA was slightly increased at $6 \mathrm{~h}$ after Wnt3a treatment; however, we did not observe significant a

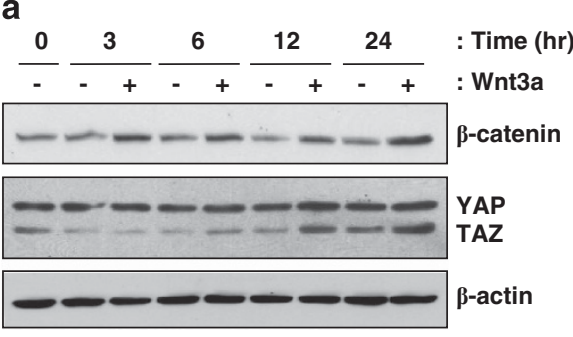

b

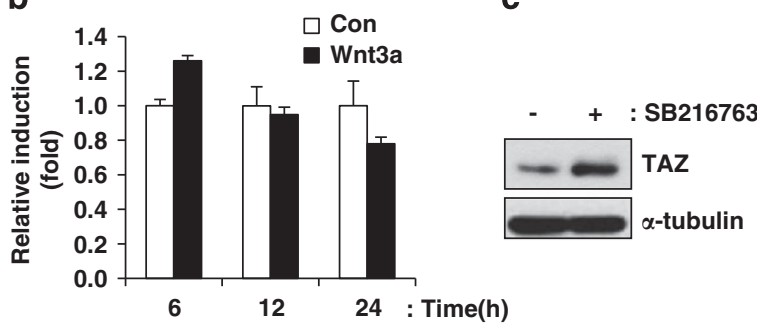

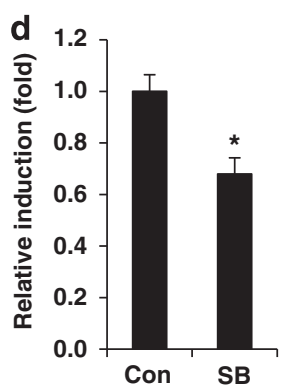

e

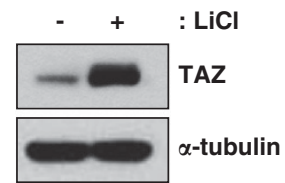

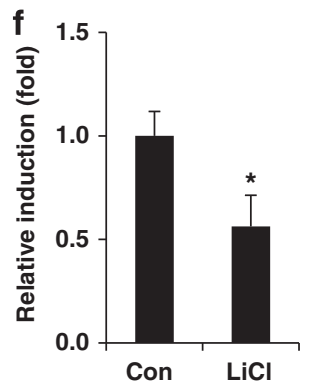

g

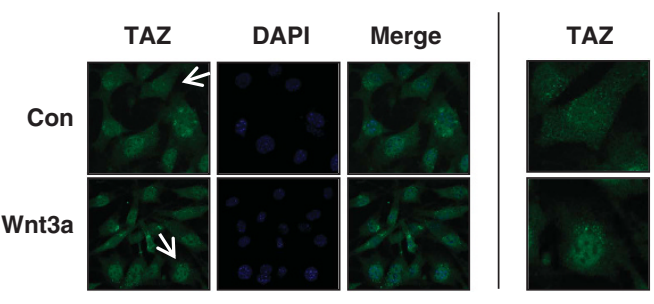

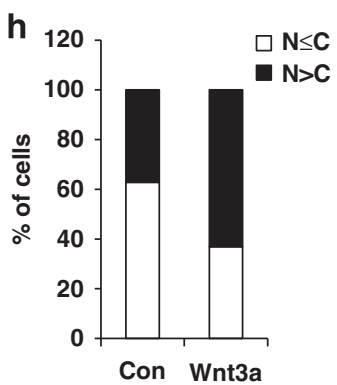

i

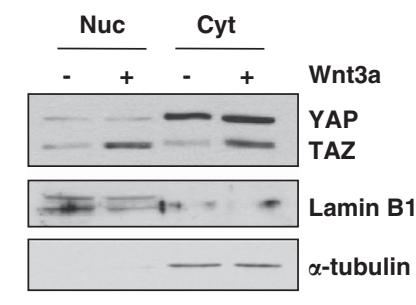

j

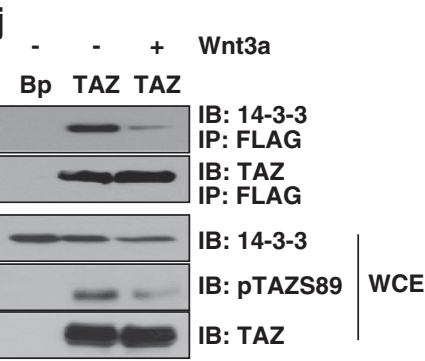

Figure 1 Wnt3a increases TAZ expression and stimulates the nuclear localisation of TAZ. (a) Proliferating C3H10T1/2 cells were serum-depleted for $16 \mathrm{~h}$ and subsequently incubated in Wnt3a-conditioned medium, which was derived from Wnt3a-secreting $\mathrm{L}$ cells. At the indicated times, cell lysates were prepared and the expression levels of $\beta$-catenin and TAZ were analysed by western blot analysis. The expression of $\beta$-actin was analysed as a loading control. ' - ' indicates control L cells condition medium. (b) The mRNAs in (a) were isolated, and TAZ expression was analysed by quantitative RT-PCR (qRT-PCR). (c) A GSK3 $\beta$ inhibitor increases TAZ expression. C3H10T1/2 cells were incubated with $5 \mu \mathrm{M}$ of SB216763 (SB) for $24 \mathrm{~h}$. Total cell lysates were then collected, and the level of TAZ was analysed by western blot analysis. The level of $\beta$-actin was analysed as a loading control. (d) Total RNA was isolated from the cells in (c). The expression of TAZ was analysed by qRT-PCR. ${ }^{*} P<0.05$ by Student's t-test. (e) Growing C3H10T1/2 cells were incubated with $25 \mathrm{mM} \mathrm{LiCl}$, and, $24 \mathrm{~h}$ later, the level of TAZ was analysed by western blot analysis. (f) Total RNAs were isolated from the cells in (e). The expression of TAZ was analysed by qRT-PCR. ${ }^{\star} P<0.05$ by Student's $t$-test. (g) The cellular localisation of TAZ was analysed by immunocytochemistry. Experiments were performed using the protocols described in the Materials and Methods section. DAPI was used for the detection of nuclei. Pictures in right side are the magnified ones from cells indicated by arrow in left side. (h) Quantitative analysis of TAZ localisation. The cellular distribution of TAZ was analysed based on whether TAZ levels were higher in the nucleus $(\mathrm{N}>\mathrm{C})$, higher in the cytoplasm or evenly distributed between the nucleus and cytoplasm $(\mathrm{N} \leq \mathrm{C})$. The percentage of cells in each category was determined after observing cells in five different fields of (g). (i) C3H10T1/2 cells were treated with Wnt3a-conditioned medium for $24 \mathrm{~h}$, and the cell lysates were prepared and fractionated into cytosol (Cyt) and nuclear (Nuc) extracts according to the experimental methods. The expression of TAZ was analysed by western blot analysis. (j) Wnt3a decreases 14-3-3 binding to TAZ. Control (Bp) and FLAG-TAZ-overexpressing C3H10T1/2 cells (TAZ) were incubated with Wnt3a-conditioned medium, and, after 24 h, cell lysates were prepared for the analysis of TAZ phosphorylated at serine 89 and its binding with 14-3-3. Whole-cell extracts (WCE) were analysed using antibodies against 14-3-3, TAZ and phospho-TAZ (at serine 89). The cell extracts were immunoprecipitated with an anti-FLAG antibody, and the immune complexes were immunoblotted with anti-TAZ and 14-3-3 antibodies 
induction of TAZ mRNA at 12 and $24 \mathrm{~h}$, suggesting that posttranscriptional regulation of $T A Z$ is important for the induction of TAZ after Wnt3a treatment.

Canonical Wnts stabilise $\beta$-catenin through the inactivation of GSK3. Thus, inhibition of GSK3 mimics stimulation of canonical Wnt signalling. It was shown that GSK3 is important for the proteolytic degradation of TAZ. ${ }^{29}$ Thus, we studied whether GSK3 is important for TAZ regulation in C3H10T1/2 cells. The expression of TAZ was monitored in the presence of the GSK3 inhibitor SB216763. As shown in Figure 1c, the inhibitor increased TAZ expression. However, the RNA expression of TAZ was not induced after SB216763 treatment (Figure 1d). These results suggest that GSK3 regulates TAZ at the post-transcriptional level. The induction of TAZ by GSK3 inhibition was also studied via treatment with $\mathrm{LiCl}$, a GSK3 inhibitor with broad specificity. Treatment with $\mathrm{LiCl}$ also increased TAZ protein expression (Figure 1e) but not mRNA expression (Figure 1f), suggesting that the inhibition of GSK3 affects TAZ expression at the post-transcriptional level.

Wnt signalling stabilises $\beta$-catenin and stimulates its nuclear localisation. Thus, we examined whether Wnt3a stimulates the nuclear localisation of TAZ as it does with $\beta$-catenin. Immunocytochemical analysis was employed in these experiments. As shown in Figure 1g, increased TAZ expression intensity was observed in the nuclei of Wnt3atreated cells compared with control cells. Quantification revealed that Wnt3a stimulates the nuclear localisation of TAZ (Figure 1h). These results were confirmed with a biochemical study. The lysates of Wnt3a-treated cells were separated into nuclear and cytosolic fractions. An increased level of TAZ was observed in the nucleus of Wnt3a-treated cells compared with control cells, suggesting that Wnt3a facilitates the nuclear localisation of TAZ-like $\beta$-catenin (Figure 1i).

To see whether the TAZ induction by Wnt3a occurs in a cell type-dependent manner, human MSCs, NIH3T3 and mouse embryonic fibroblast (MEF) were incubated with Wnt3a, and we observed that TAZ was induced by Wnt3a in these cells, showing that TAZ induction by Wnt3a is not a cell type-specific response (Supplementary Figure 1).

The nuclear localisation of $T A Z$ is regulated by its phosphorylation. ${ }^{1}$ Serine 89 -phosphorylated TAZ at serine 89 is recognised by 14-3-3 proteins, and these proteins sequester TAZ in the cytosol. ${ }^{1}$ Thus, we studied whether the phosphorylation status of TAZ is regulated by Wnt3a. FLAG-tagged TAZ-overexpressing C3H10T1/2 cells were incubated in Wnt3a-conditioned medium, and we observed that the level of serine 89-phosphorylated TAZ was decreased in the presence of Wnt3a (Figure 1j). Next, because the phosphorylation of TAZ at serine 89 is important for 14-3-3 binding, the 14-3-3 binding efficiency was investigated. TAZ-expressing cell lysates were immunoprecipitated with an anti-FLAG antibody, and the bound 14-3-3 proteins were analysed. The results indicated that Wnt3a significantly reduced $14-3-3$ binding to TAZ, suggesting that increased nuclear localisation of TAZ after Wnt3a stimulation is induced by increased dephosphorylation of TAZ at serine 89 and decreased 14-3-3 binding. Taken together, these results indicate that Wnt3a stimulates TAZ expression and nuclear localisation.
Wnt3a inhibits proteolytic cleavage of TAZ by producing dephosphorylated TAZ. The protein stability of TAZ is regulated by its phosphorylation. In the $\mathrm{N}$ - and $\mathrm{C}$-terminal regions of $\mathrm{TAZ}$, there are phosphodegron motifs that can be recognised by proteasomal complexes. ${ }^{30-32}$ The phosphorylation of the C-terminal phosphodegron motif is activated by Hippo signalling, which is activated by a high density of cells in culture. At high culture densities, TAZ is phosphorylated by Lats kinase and casein kinase $1 .^{33}$ This phosphorylation induces the interaction between TAZ and $\beta$-TrCP and stimulates the proteolytic degradation of TAZ. ${ }^{31}$ Indeed, we observed that high-density cultivation of $\mathrm{C} 3 \mathrm{H} 10 \mathrm{~T} 1 / 2$ cells decreases endogenous TAZ (Figure 2a). Next, we studied whether Wnt3a inhibits the proteolytic degradation of TAZ induced by a high culture density. We found that TAZ degradation was significantly inhibited in the presence of Wnt3a, whereas treatment with MG132, protease inhibitor, blocked the proteolytic degradation of TAZ in high-density culture condition (Figure $2 \mathrm{~b}$ ). We also found that Wnt3a increased nuclear localisation of TAZ in high-density culture condition (Supplementary Figure 2). These results suggest that Wnt signalling blocks the proteolytic degradation of TAZ triggered by its phosphorylation. Thus, we studied whether the phosphorylation of TAZ at serine 306 and 309, which are located in the C-terminal phosphodegron motif and phosphorylated by Lats kinase and CK1, is inhibited by Wnt3a. For the experiment, C3H10T1/2 cell lysates were prepared and immunoprecipitated with an anti-TAZ antibody in the presence or absence of Wnt3a. The immune complexes were analysed with a phosphospecific antibody (Supplementary Figure $3 \mathrm{~B}$ ) that recognises phosphorylated serines at positions 306 and 309 of TAZ. Indeed, Wnt3a significantly decreased the proteolytic degradation triggered by phosphorylation of TAZ, indicating again that Wnt3a inhibits the proteolytic pathway induced by phosphorylation (Figure 2c).

Next, we studied whether Wnt3a inhibits ubiquitinationmediated proteolytic degradation of TAZ. TAZ and ubiquitin expression plasmids were introduced into cells, TAZ was immunoprecipitated and in vitro ubiquitinated bands were analysed with the immune complexes. In Figure 2d, decreased ubiquitinated TAZ was observed in Wnt3a-treated cells. Thus, these results suggest that Wnt3a stabilises TAZ by blocking Hippo signal-mediated proteolytic degradation of TAZ.

Next, we studied whether Wnt3a affects the stability of phosphodegron motif mutants of TAZ. TAZ wild-type, TAZ-Ser306/309Ala (TAZS306,309A) and TAZ-Ser58/62Ala (TAZS58,62A) mutants expressing stable cell lines were prepared using retrovirus. The expression of TAZ was analysed in the absence or presence of Wnt3a. In Figure $2 e$, the expression of TAZ mutants was significantly increased compared with the one of TAZ wild type in the absence of Wnt3a. This was caused by post-transcriptional regulation because the levels of TAZ mRNA expression were not different between wild type and mutants (Figure 2f). Thus, the results show that the phosphodegron motifs are important sites for TAZ expression. In the presence of Wnt3a, wild-type TAZ and TAZS58,62A mutant expression was increased compared with their expression in the absence of Wnt3a, 


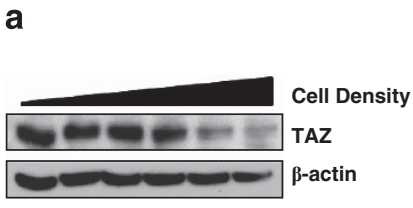

b

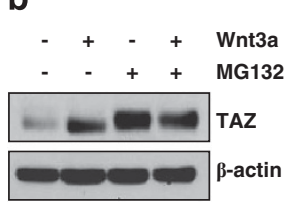

d

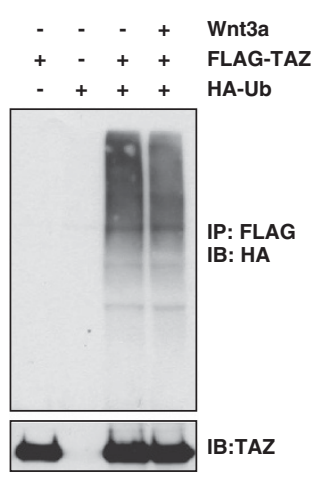

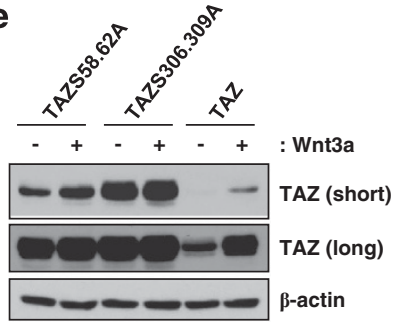

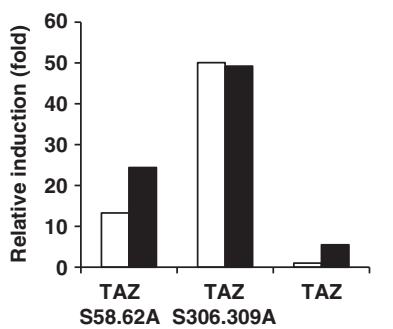

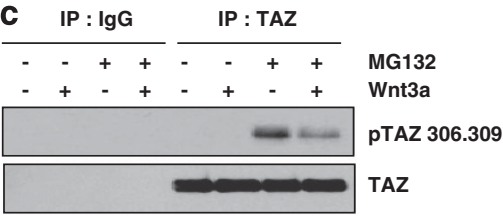

f

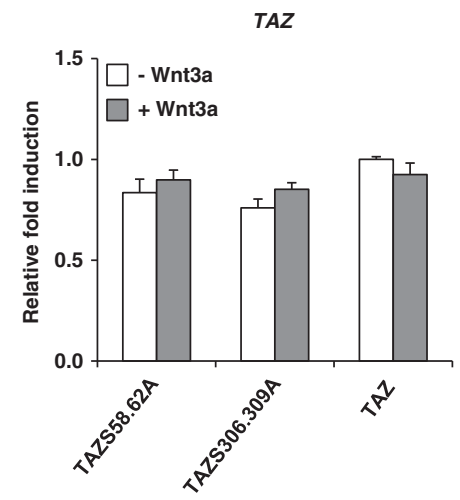

Figure 2 Wnt3a induces dephosphorylation of TAZ. (a) Increasing amounts of C3H10T1/2 cells $\left(0.1,0.2,0.5,1,2\right.$ and $3 \times 10^{6}$ cells/well) were plated onto six-well plates, and, after $24 \mathrm{~h}$, TAZ expression was analysed by western blot analysis. The expression of $\beta$-actin was analysed as a loading control. (b) $\mathrm{C} 3 \mathrm{H} 10 \mathrm{~T} 1 / 2 \mathrm{cells}$ were plated at a high density $\left(3 \times 10^{6}\right.$ cells/each well) and incubated with Wnt3a-conditioned medium for $24 \mathrm{~h}$ in the presence or absence of $10 \mu \mathrm{M} \mathrm{MG132}$. Next, cell lysates were prepared, and TAZ expression was analysed. (c) The cell lysates in (b) were immunoprecipitated with an anti-TAZ antibody (BD Biosciences, cat\#560235). TAZ phosphorylated at serine 306 and 309 and unphosphorylated TAZ were analysed by western blot analysis. (d) FLAG-TAZ and HA-Ubiquitin expression plasmids were transfected into 293T cells and incubated with Wnt3a-conditioned medium in the presence of $10 \mu \mathrm{M} \mathrm{MG132}$ for $12 \mathrm{~h}$. Subsequently, the cell lysates were immunoprecipitated with anti-FLAG agarose beads, and the precipitated proteins were separated on SDS-PAGE and analysed with an anti-HA antibody for detection of ubiquitinated TAZ. Introduced TAZ was analysed by western blot analysis. (e) FLAG-tagged TAZ wild-type, TAZS306,309A, and TAZS58,62A mutants expressing C3H10T1/2 cells were prepared by retrovirus. The stable cell lines were incubated in the presence or absence of Wnt3a. At $24 \mathrm{~h}$ after Wnt3a treatment, TAZ expression was analysed by western blot analysis. The expression of $\beta$-actin was analysed as a loading control. The bottom panel indicates the expression levels of TAZ analysed by a densitometer. Short and long indicate film exposure time. (f) The mRNAs in (e) were isolated, and TAZ expression was analysed by qRT-PCR

but the expression of TAZS306,309A was not increased. The results suggest that the expression of TAZS306,309A mutant is not affected by Wnt3a signal and the de-phosphorylation of serine 306 and 309 is important for Wnt3a-induced TAZ stabilisation.

PP1A is a mediator of Wnt3a-induced TAZ stabilisation. PP1A is a phosphatase that can interact with TAZ and dephosphorylate the sites that are important for 14-3-3 binding and $\beta$-TrCP binding. ${ }^{34}$ Thus, Wnt3a may modulate the phosphorylation status of TAZ and block proteolytic degradation through PP1A. To understand the mechanism of TAZ stabilisation after Wnt3a stimulation, we studied whether TAZ is regulated by PP1A in the presence of a Wnt signal. For the experiment, Wnt3a-treated cells were prepared, and the endogenous interaction between TAZ and PP1A was analysed. As shown in Figure $3 a$, ectopically expressed FLAG-tagged TAZ interacted with endogenous PP1A and Wnt3a significantly increased this interaction. Also in Figure $3 b$, endogenous TAZ physically interacted with PP1A and further increased interaction was observed in the presence of Wnt3a. The results suggest that PP1A may facilitate the de-phosphorylation of TAZ in the presence of Wnt3a. Next, to further study the effect of PP1A on TAZ expression, we depleted PP1A using small interfering RNAs (siRNAs) (Figure $3 c$ ). We observed that PP1A could be depleted by three different siRNAs, and this depletion decreased the expression of TAZ (Figure $3 c$ ), suggesting that PP1A is a mediator of Wnt3a-induced TAZ stabilisation. Next, to further study the effect of PP1A on Wnt3a-mediated TAZ expression, the cells were treated with okadaic acid, a PP1A inhibitor, in the presence of Wnt3a. We observed that okadaic acid significantly inhibited TAZ expression (Figure $3 d$ ). This inhibition was regulated at the posttranscriptional level because TAZ mRNA expression was not inhibited after okadaic acid treatment (Figure 3e). We also studied whether SB216763- or LiCl-induced TAZ is regulated by okadaic acid. As shown in Figures $3 f$ and $g$, okadaic acid blocked SB216763- or LiCl-induced TAZ expression. Thus, these results suggest that PP1A is an important regulator of Wnt3a-mediated TAZ expression. In this experiment, treatment with SB216763 or LiCl did not increase TAZ mRNA levels, indicating that post-transcriptional regulation of $T A Z$ is responsible for the change in TAZ expression (data not shown).

Wnt3a stimulates TAZ-mediated osteogenic differentiation. Next, we studied whether Wnt3a induces TAZ during 
a

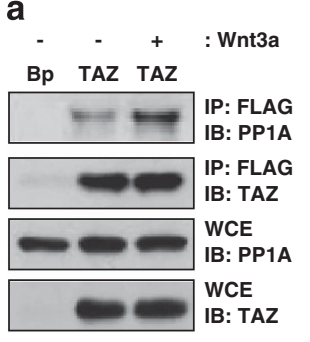

b

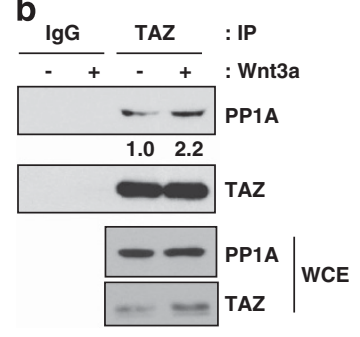

c

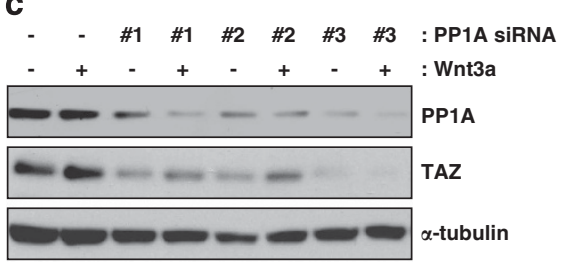

f
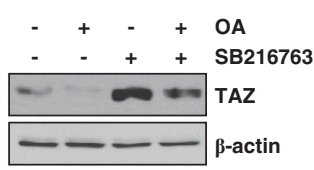

g - + - + OA

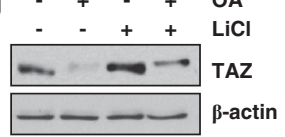

Figure 3 Increased TAZ stability through Wnt3a signalling is induced by PP1A. (a) FLAG-tagged TAZ-expressing C3H10T1/2 cells were incubated in the presence or absence of Wnt3a, and the cell lysates were immunoprecipitated with an anti-FLAG antibody. TAZ bound to PP1A was analysed by western blot analysis. WCE indicates whole-cell lysates. IP and IB indicate immunoprecipitation and immunoblot, respectively. (b) C3H10T1/2 cells were incubated in the presence or absence of Wnt3a, and the cell lysates were immunoprecipitated with an immunoglobulin $\mathrm{G}(\mathrm{IgG})$ or anti-TAZ antibody. Endogenous TAZ bound to PP1A was analysed by western blot analysis. WCE indicates whole-cell lysates. (c) PP1A depletion leads to a decreased level of TAZ. C3H10T1/2 cells were transfected with $100 \mu$ mol of mouse PP1A siRNA \#1, \#2, and \#3 for $24 \mathrm{~h}$ and subsequently incubated in Wnt3a-conditioned medium for $24 \mathrm{~h}$. Next, cell lysates were prepared, and PP1A and TAZ expression levels were examined by western blot analysis. (d) Okadaic acid (OA) decreases TAZ expression. C3H10T1/2 cells were incubated in Wnt3a-conditioned medium with or without $50 \mathrm{ng} / \mathrm{ml}$ okadaic acid. After $48 \mathrm{~h}$, the cells were lysed, and the cell lysates were analysed by western blot analysis. (e) The mRNAs in (d) were isolated, and TAZ expression was analysed by qRT-PCR. ${ }^{*} P<0.01$ by Student's $t$-test. (f) Okadaic acid suppresses SB216763-induced TAZ expression. The C3H10T1/2 cells were incubated with $5 \mu \mathrm{M}$ SB216763 for $24 \mathrm{~h}$, and $50 \mathrm{ng} / \mathrm{ml}$ okadaic acid was then added and incubated for $4 \mathrm{~h}$. The cell lysates were analysed by western blot analysis. (g) Okadaic acid suppresses LiCl-induced TAZ expression. C3H10T1/2 cells were incubated with $25 \mathrm{mM} \mathrm{LiCl} \mathrm{for} 24 \mathrm{~h}$, and $50 \mathrm{ng} / \mathrm{ml}$ okadaic acid was then added and incubated for $4 \mathrm{~h}$. The cell lysates were subjected to western blot analysis

osteoblast differentiation. C3H10T1/2 cells were incubated in osteogenic differentiation media with or without Wnt3a. To determine the osteogenic potential of Wnt3a, alkaline phosphatase activity was investigated. As shown in Figure $4 \mathrm{a}$, increased alkaline phosphatase activity was observed in Wnt3a-treated cells. We also observed increased expression levels of osteogenic marker genes including Osterix (OSX), MSX2, Osteocalcin (OC) and RunX2 in Wnt3a-treated cells by qRT-PCR (Figure 4b). The expression of $T A Z$ was increased by 1.6-fold compared with the control (Figure $4 b$ ), and an increase in TAZ protein was also observed in the presence of Wnt3a during osteoblast differentiation (Figure 4c), indicating that Wnt3a stimulates TAZ expression during osteoblast differentiation. We also analysed the osteogenic effect of Wnt3a in human MSCs and observed that Wnt3a increased TAZ and osteogenic marker genes including OC, MSX2 and Runx2 during osteogenic differentiation (Supplementary Figure 4).

Next, to examine the role of TAZ in Wnt3a-mediated transcriptional activation of osteoblast differentiation, we studied whether Wnt3a stimulates TAZ to upregulate Runx2-mediated gene transcription using Runx2-binding sites linked to a luciferase reporter construct (6XOSE2-Luc). As shown in Figure 4d, TAZ significantly activated Runx2mediated gene transcription in the presence of Wnt3a. It appeared that this activation was derived from the increased nuclear localisation of TAZ and its interaction with Runx2 in the presence of Wnt3a. Indeed, we observed that the physical interaction of TAZ and Runx2 was significantly increased in the presence of Wnt3a (Figure 4e).

Next, to study whether TAZ is important for Runx2mediated gene transcription after Wnt3a treatment, the reporter gene was introduced in control and TAZ knockdown cells (Supplementary Figure 5A) and Wnt3a-induced Runx2 reporter gene activity was analysed. In Supplementary Figure 5B, Wnt3a significantly activated Runx2 transcriptional activity, but the increased Runx2 activity by Wnt3a was not observed in TAZ knockdown cells. Thus, the results suggest that TAZ is an important factor for Wnt3a-induced ostegeogenic marker gene transcription.

Next, to study whether Wnt3a increases the localisation of TAZ to the Runx2-binding site in the osteocalcin and Runx2 promoters, we performed a chromatin immunoprecipitation assay. As shown in Figure 4f, TAZ was significantly recruited onto the osteocalcin and Runx2 promoters in the presence of Wnt3a. Taken together, these results suggest that Wnt3a activates Runx2-mediated osteoblastic gene transcription via recruitment of TAZ to the promoter of osteoblast marker genes.

To further understand the role of TAZ in Wnt3a signalling, endogenous TAZ was depleted using a TAZ-specific shRNA in $\mathrm{C} 3 \mathrm{H} 10 \mathrm{~T} 1 / 2$ cells, and the cells were incubated with osteogenic differentiation media with or without Wnt3a. As shown in Figure $5 a, T A Z$ depletion was observed in cells transformed with TAZ-specific shRNA, and Wnt3a-induced TAZ expression was not observed in TAZ-depleted cells. 

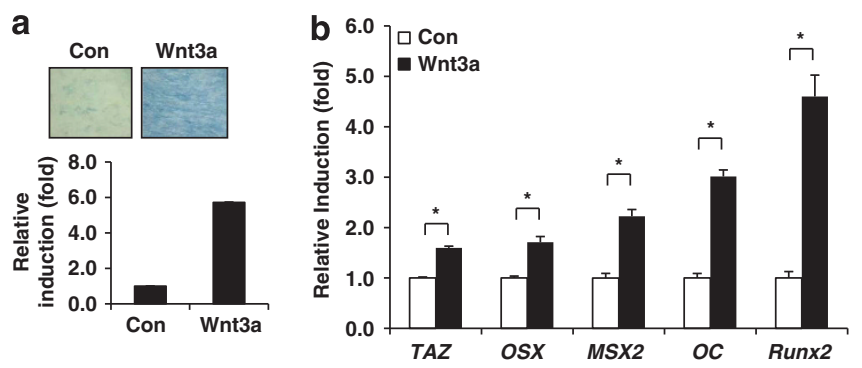

C
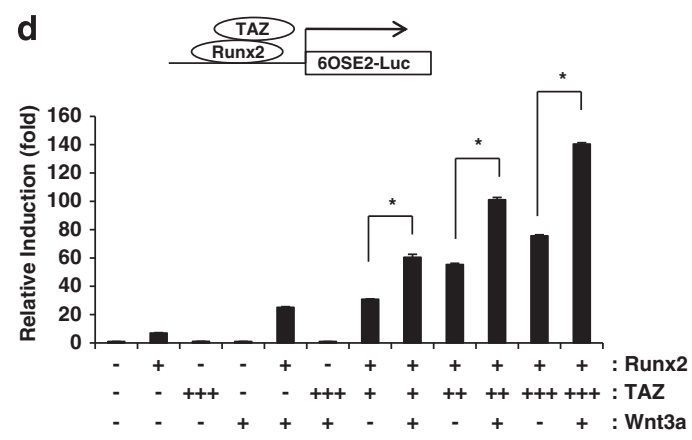

e +-++ FLAG TAZ
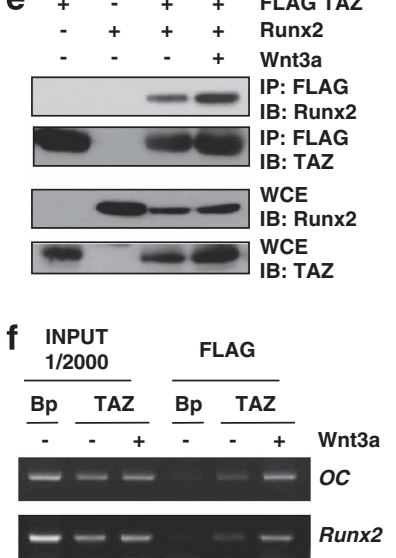

Figure 4 Wnt3a-induced osteogenic differentiation is regulated by TAZ. (a) C3H10T1/2 cells were incubated in Wnt3a-conditioned medium. At 4 days after Wnt3a treatment, alkaline phosphatase activity was analysed to determine the osteogenic potential of Wnt3a. A blue colour indicates increased alkaline phosphatase activity. (b) Total RNAs were prepared 2 days after osteoblast differentiation in the presence or absence of Wnt3a. The expression levels of the osteoblast marker genes osterix (OSX), MSX2, osteocalcin (OC), Runx2 and TAZ were analysed by qRT-PCR. ${ }^{*}$ Indicates $P<0.01$ by Student's $t$-test. (c) Increased TAZ expression was observed in Wnt3a-treated cells. At the indicated time points, cell lysates were prepared, and the expression levels of TAZ and $\beta$-actin were analysed by western blot. (d) Wnt3a stimulates TAZ-mediated Runx2 target gene activation. Luciferase reporter activity from a construct containing six copies of the Runx2-binding site (6OSE2-Luc) was measured in cell lysates $48 \mathrm{~h}$ after transfection and normalised to Renilla luciferase activity. TAZ and/or Runx2 expression plasmids were introduced into 293T cells. The luciferase activity was measured in the absence or presence of Wnt3a. An increasing number of + indicates an increasing amount of expression plasmids for transfection. ${ }^{*} P<0.05$ by Student's $t$-test. (e) 293T cells were transfected with Runx2 and/or FLAG-tagged TAZ expression plasmids and incubated with Wnt3a-conditioned medium for $24 \mathrm{~h}$. Whole-cell extracts (WCE) were precipitated with FLAG-M2 agarose beads. The precipitates and WCE were analysed by immunoblot with antibodies against TAZ and Runx2. (f) Chromatin immunoprecipitation of TAZ with the endogenous $O C$ and Runx2 promoters in response to Wnt3a stimulation. Stable FLAG-tagged TAZ-expressing C3H10T1/2 cells were treated for 2 days with Wnt3a, and the immunoprecipitates obtained with an anti-FLAG antibody were analysed for OC and Runx2 promoter occupancy by PCR

As shown in Figure 5b, Wnt3a induced alkaline phosphatase activity in control cells, but TAZ-depleted cells showed significantly reduced activity (an approximate $50 \%$ reduction compared with control cells). In addition, the expression of the osteogenic marker gene osteocalcin and TAZ was significantly reduced in TAZ-depleted cells (Figure $5 \mathrm{c}$ ).

Next, TAZ-overexpressing cell lines were prepared by retrovirus in TAZ knockdown C3H10T1/2 cells and recovered TAZ expression in TAZ-depleted cells was observed in Figure 6a. The TAZ overexpression increased osteogenic potential of cells, which was decreased by TAZ depletion, as evidenced by alkaline phosphatase activity (Figure 6b). Next, Wnt3a-mediated osteogenic differentiation activity was analysed in control, TAZ-depleted and TAZ-introduced cells. In Figure 6c, the TAZ-introduced cells showed increased $O C$, OSX, MSX2 and Runx2 expression compared with TAZdepleted cells or control cells. Also further significant induction was observed in the presence of Wnt3a (Figure 6c). Thus, these results suggest that TAZ has an important role in Wnt3a-mediated osteoblast differentiation.

\section{Discussion}

In this study, we showed that TAZ, a Hippo effector, is an important mediator in Wnt3a-stimulated osteoblast differentiation. The expression level of TAZ was increased in the presence of $\mathrm{Wnt} 3 \mathrm{a}$, and this increased expression stimulated osteoblast differentiation (see Supplementary Figure 6). Depletion of TAZ did not affect Wnt3a-induced osteogenic differentiation. In addition, inhibition of GSK3 caused an increase in TAZ expression. The Wnt3a-induced increase in TAZ expression was a result of TAZ stabilisation. Wnt3a produced TAZ that was dephosphorylated at serine 89, 306 and 309, whereas TAZ can be phosphorylated by the Hippo signalling components Lats and CK1 to induce proteolytic degradation. ${ }^{31}$ We observed that Wnt3a increased the physical interaction between TAZ and PP1A and facilitated the dephosphorylation of TAZ, which blocks the proteolytic degradation of TAZ (Figure 3). We also observed that the high-density culture-induced proteolytic degradation of TAZ was 
a

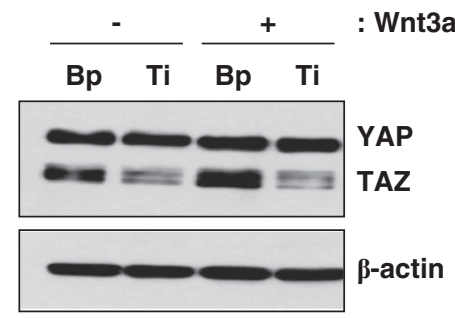

b
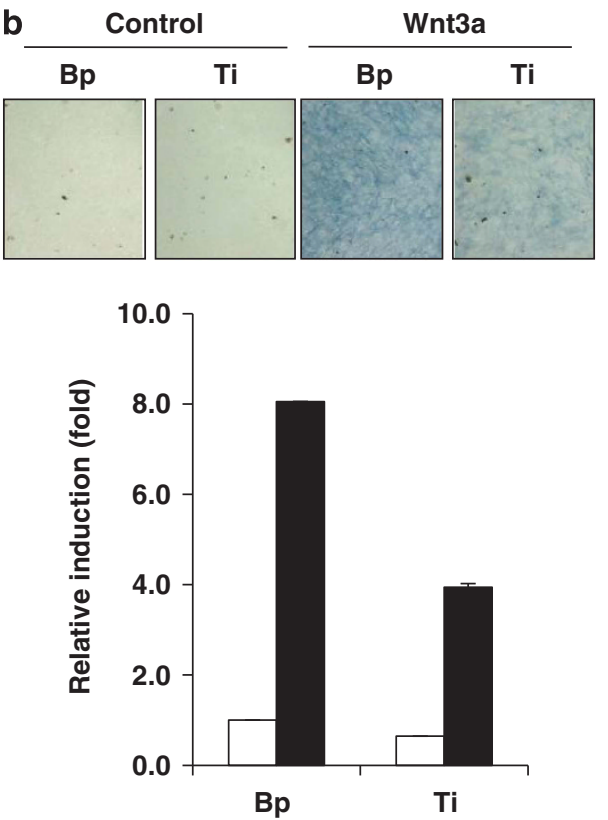

C

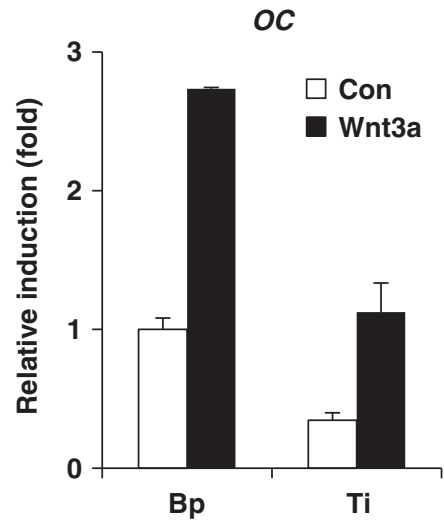

$T A Z$

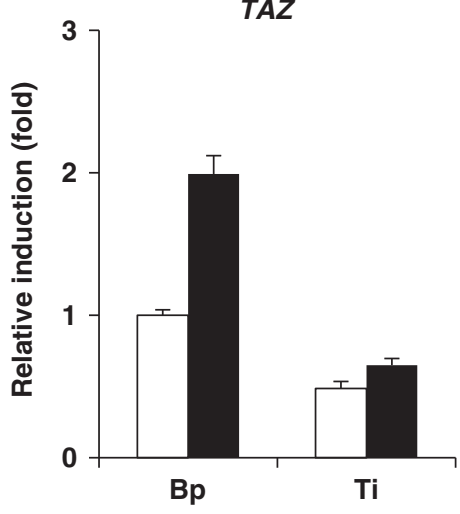

Figure 5 (a) C3H10T1/2 cells were infected with TAZ shRNA-producing retroviruses, and stable TAZ-depleted cells (Ti) were prepared. Retrovirus derived from a control vector was used for making control cells (Bp). The cells were induced to osteoblastic differentiation with or without Wnt3a-conditioned media. At the indicated time points, total cell lysates were prepared, and the level of TAZ was analysed by western blot. (b) Alkaline phosphatase activity was analysed by cellular staining and enzymatic assays. At 4 days after differentiation, the cells in (a) were stained with an alkaline phosphatase staining solution. For activity quantification, the differentiated cells were lysed, and the enzymatic activity of alkaline phosphatase was analysed as described in the Materials and Methods section. (c) Total RNA was isolated from the cells in (b). The expression of $O C$ and $T A Z$ was analysed by qRT-PCR at 2 days after differentiation

a

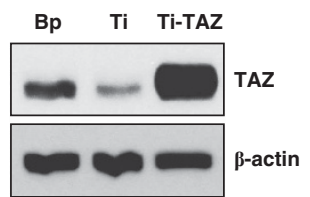

b

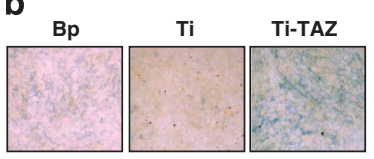

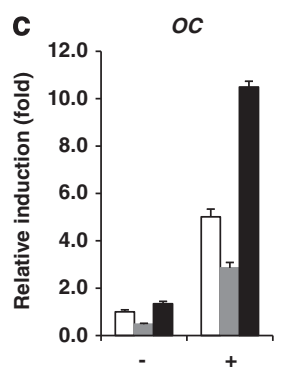
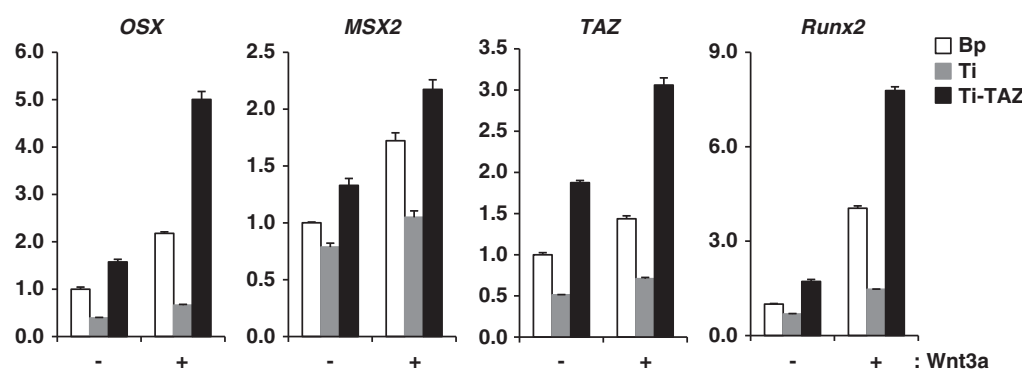

Figure 6 TAZ overexpression induces osteogenic genes transcription, which was decreased by TAZ depletion. (a) Re-introduction of TAZ in C3H10T1/2 cells was assessed by a retrovirus producing Flag-TAZ. Bp and Ti indicate vector control cells and TAZ knockdown cells, respectively. Ti-TAZ indicates Flag-TAZ introduced TAZ knockdown cells. TAZ expression was analysed by western blot. (b) Bp, Ti and Ti-TAZ cells were differentiated in osteogenic differentiation medium. At 2 days after differentiation, alkaline phosphatase activity was analysed to determine the osteogenic potential of each cells. A blue colour indicates increased alkaline phosphatase activity. (c) The cells in (a) were induced to osteoblastic differentiation with or without Wnt3a-conditioned media. At 2 days after differentiation, total RNAs were collected from the cells, and OC, OSX, MSX2, TAZ and Runx2 expression levels were analysed by qRT-PCR. Their relative expression was determined after normalization to the GAPDH level 
inhibited by Wnt3a (Figure 2). These results suggest that Hippo signalling can be suppressed by Wnt signalling.

Recent studies have shown that Hippo signalling regulates Wnt signalling. Cytoplasmic TAZ and YAP interact with Dishevelled (DVL) and inhibit Wnt signalling by inhibiting the CK $1 \delta / \varepsilon$-mediated phosphorylation of DVL, interfering with the nuclear localisation of nuclear $\beta$-catenin. ${ }^{35}$ Imajo et al. ${ }^{36}$ have shown that phosphorylated YAP binds to $\beta$-catenin and suppresses the nuclear translocation of $\beta$-catenin in the cytoplasm. Muscle-specific conditional knockout of Salvador, which activates Lats kinase, reduced YAP phosphorylation and elevated cardiomyocyte proliferation with increased nuclear accumulation of $\beta$-catenin and expression of $\beta$-catenin target genes. ${ }^{37}$ YAP was shown to interact with $\beta$-catenin and activate target genes of Wnt signalling. ${ }^{37}$ Another group showed that YAP stimulated insulin-like growth factor signalling following activation of AKT and inhibition of glycogen synthase kinase $3 \beta$. This produced increased $\beta$-catenin, which stimulated Wnt target genes. ${ }^{38}$ These results suggest that Hippo signalling antagonises Wnt signalling. However, it was recently shown that Wnt signalling regulates the Hippo pathway. Konsavage et al. ${ }^{39}$ have shown that the Wnt signal stimulates YAP expression in colorectal carcinoma cells. It was also shown that the Wnt signal regulates TAZ activity, although it was revealed that this regulation is independent of Hippo signalling. ${ }^{29}$ Our results provide evidence that Wnt signalling stabilises TAZ but not YAP in the cellular proliferation and differentiation, suggesting that bidirectional regulatory signalling occurs between the Hippo and Wnt pathways, in which a selective Hippo effector is regulated.

TAZ and YAP have similar protein structures containing WW domain and coiled-coil domain and showed redundant cellular functions including cell proliferation and migration. However, functional differences between the two proteins have been also suggested previously. For example, the phenotypes of TAZ and YAP knockout mice are different; YAP knockout mice are embryonic lethal, whereas TAZ knockout mice are viable, although there is some difficulty in obtaining viable mice because of a perinatal lethal phenotype. ${ }^{40,41}$ Also, during mesenchymal stem cell differentiation, TAZ stimulates osteogenic differentiation through Runx2 activation, whereas YAP suppresses Runx2-mediated gene transcription, in which src-mediated YAP phosphorylation facilitates its interaction with Runx2 for the suppression of osteocalcin expression. ${ }^{42}$ These results indicate that TAZ and YAP have an opposite role in the Runx2-mediated gene transcription. Thus, it stands to reason that Wnt3a activates TAZ, not YAP, for the osteogenic differentiation. In relation with this, an interesting question is how two proteins can be differentially regulated by Wnt signalling. At present, the mechanism for this regulation is not clear, although it was shown that PP1A is a phosphatase that dephosphorylates TAZ but not YAP. ${ }^{34}$ Thus, it appears that the specific interaction between TAZ and PP1A is responsible for the stabilisation of TAZ but not YAP after Wnt3a stimulation.

TAZ-depleted cells show decreased osteogenic potential compared with wild-type cells in the presence of Wnt3a, suggesting that, similar to $\beta$-catenin, TAZ is a signalling mediator in Wnt3a-induced osteoblast differentiation.
This raises the question of whether TAZ has a unique role in Wnt signalling that is different from that of $\beta$-catenin in bone development. Previously, it was shown that osteoblastspecific depletion of $\beta$-catenin leads to decreased bone mass caused by an increase in osteoclastogenesis without an effect on osteogenesis. ${ }^{26-28}$ However, regulators upstream of $\beta$-catenin such as Wnt ligands and receptors have demonstrated increased osteogenic potential. For example, osteoblast-specific Wnt10b transgenic mice show increased bone formation concurrent with increased osteoblast cell number, and genetic alteration of Lrp5 regulates bone formation. ${ }^{23,43-45}$ These results suggest that there are $\beta$-catenin-independent pathways for Wnt signal-induced bone formation in vivo. Thus, our results suggest a model in which TAZ might be a $\beta$-catenin-independent Wnt signal mediator during bone formation in vivo, although further studies are required for clear understanding. At this point, it is notable that TAZ knockout mice show decreased ossification, ${ }^{46}$ and osteoblast-specific TAZ transgenic mice show increased bone mass without alterations in osteoclast activity. ${ }^{47}$ Taken together, our results reveal that TAZ is stabilised by Wnt3a and regulates Wnt3a-stimulated osteoblast differentiation, suggesting that TAZ is an effector protein of Wnt3a signalling.

\section{Materials and Methods}

Cell culture and osteoblast differentiation. C3H10T1/2 cells were maintained in Dulbecco's modified Eagle's medium (DMEM) supplemented with $10 \%$ FBS (growth medium) and antibiotics. To induce osteoblast differentiation, C3H10T1/2 cells were seeded onto 24-well culture plates at a density of $2 \times 10^{4}$ cells $/ \mathrm{cm}^{2}$, and, $48 \mathrm{~h}$ later, the culture medium was replaced with differentiation medium containing growth medium, $50 \mu \mathrm{g} / \mathrm{ml}$ ascorbic acid and $10 \mathrm{mM}$ $\beta$-glycerophosphate (differentiation medium) for 2-4 days.

Wnt3a-conditioned media. L-Wnt3a cells (Wnt3a-expressing cells) and $\mathrm{L}$ cells (control) were cultured in DMEM with 10\% FBS and antibiotics. To obtain $\mathrm{L}$ or L-Wnt3a-conditioned media, cells were seeded onto $100-\mathrm{mm}$ culture dishes, and, $24 \mathrm{~h}$ later, the culture medium was replaced with DMEM containing 10\% FBS and antibiotics. After 2 days, media from cultivated $L$ and L-Wnt3a cells was collected and stored at $4{ }^{\circ} \mathrm{C}$. For the Wnt3a treatment, growth medium containing $\mathrm{L}$ or $\mathrm{L}-\mathrm{Wnt} 3 \mathrm{a}$ conditioned medium was used.

Alkaline phosphatase staining and activity assay. Differentiated osteoblast cells were washed twice with ice-cold PBS, fixed with $3.7 \%$ formaldehyde at room temperature for $10 \mathrm{~min}$, rinsed once with ice-cold PBS and stained with staining reagents $(0.1 \mathrm{mg} / \mathrm{ml}$ naphthol AS-MX phosphate (SigmaAldrich, St. Louis, MO, USA), 0.5\% N,N-dimethylformamide (Sigma-Aldrich), $2 \mathrm{mM}$ $\mathrm{MgCl}_{2}, 0.6 \mathrm{mg} / \mathrm{ml}$ Fast Blue BB salt (Sigma-Aldrich) in $0.1 \mathrm{M}$ Tris- $\mathrm{HCl}(\mathrm{pH} 8.8)$ ) for $20 \mathrm{~min}$. Staining was followed by three washes with distilled water. For the alkaline phosphatase activity assay, differentiated cells were washed with PBS, sonicated in assay buffer (25 mM HEPES (pH 7.6), 0.1\% Triton X-100, 0.9\% NaCl) and centrifuged at 13000 r.p.m. for $10 \mathrm{~min}$. The enzymatic reaction was started by mixing $50 \mu \mathrm{l}$ of cell lysates with $200 \mu \mathrm{l}$ of ALP assay solution (p-nitrophenylphosphate substrate solution (Sigma-Aldrich)) in a 96-well plate. The plate was incubated at $37^{\circ} \mathrm{C}$ for $60 \mathrm{~min}$, and $50 \mu \mathrm{l}$ of stop solution $(3 \mathrm{M} \mathrm{NaOH})$ was then added to terminate enzymatic reaction. The absorbance related to ALP enzyme activity was measured at $405 \mathrm{~nm}$ in a microplate reader.

Luciferase reporter activity assay. Cells (293T) were plated at a density of $1.5 \times 10^{5}$ cells/well in 24-well culture plates and transfected with 6OSE2-luciferase reporter, Runx2 and TAZ expression vectors using the X-tremeGENE 9 DNA Transfection Reagent (Roche, Indianapolis, IN, USA). After $24 \mathrm{~h}$, the culture medium was replaced with growth medium containing control or Wnt3a-conditioned medium. After $24 \mathrm{~h}$, the cells were lysed with passive lysis buffer (Promega, Madison, WI, USA) for $20 \mathrm{~min}$, and luciferase reporter activity was assessed using the Luciferase Assay System (Promega). 
Western blot analysis. Total cell lysates were separated on an SDS-PAGE gel and transferred to a PVDF membrane (Millipore, Billerica, MA, USA). The membranes were incubated with the following antibodies: anti-TAZ/YAP, ${ }^{2}$ antiLamin B1 (Santa Cruz Biotechnology, Dallas, TX, USA; sc-20682), anti- $\alpha$-tubulin (Ab frontier, Seoul, Korea; LF-PA0146), anti-14-3-3 (pan 14-3-3, Santa Cruz Biotechnology, sc-629), anti-PP1A (Cell Signaling Technology, Danvers, MA, USA; \#2582), anti- $\beta$-catenin (BD Biosciences, San Jose, CA, USA; \#610153), anti-Runx2 (MBL International Corporation, Woburn, MA, USA; D130-3), anti-HA (Covance, Princeton, NJ, USA; MMS-101P) and anti- $\beta$-actin (Sigma-Aldrich, A5441).

Stable cell lines. TAZ overexpressing or TAZ-depleted stable cell lines were prepared following previously described methods. ${ }^{2}$

Quantitative real-time PCR analysis. Total RNA was isolated from cells using TRIzol reagent (Invitrogen, Carlsbad, CA, USA), and CDNAs were synthesised using M-MLV reverse transcriptase (Fermentas, Burlington, ON, Canada). The mRNA expression levels of osteocalcin (OC), TAZ, MSX2, Runx2, osterix (OSX) and GAPDH were analysed by qRT-PCR (LightCycler 480, Roche). Forward $(F)$ and reverse $(\mathrm{R})$ primer sequences were as follows:

OC-F, 5'-CTGACCTCACAGATGCCAAGC-3';

OC-R, 5' $5^{\prime}$ TGGTCTGATAGCTCGTCACAAG-3';

TAZ-F, 5'-GTCACCAACAGTAGCTCAGATC-3';

TAZ-R, 5'-GGACACTGTAGCACCCTAACCCCA-3';

MSX2-F, 5'-GCACCGTGGATACAGGAGCC- $3^{\prime}$;

MSX2-R, 5'-CTTGTGTTTCCTCAGGGTGC-3';

Runx2-F, 5'-CAACCACAGAACCACAAGTGC-3';

Runx2-R, 5'-GAACTTGTGCCCTCTGTTG-3';

OSX-F, 5'-CCCCTTGTCGTCATGGTTACAG-3';

OSX-R, 5'-AGAGAAAGCCTTTGCCCACCTA-3';

GAPDH-F, 5'-GCTTGTCATCAACGGGAAG-3';

GAPDH-R, 5'-GATGTTAGTGGGGTCTCG-3'.

Immunoprecipitation. C3H10T1/2 cells stably overexpressing FLAG-TAZ (10T-TAZ) were treated with culture medium containing $\mathrm{L}$ or $\mathrm{L}-\mathrm{Wnt} 3 \mathrm{a}$-conditioned medium. After $3 \mathrm{~h}$, the cells were collected and lysed for 20 min with $150 \mathrm{mM} \mathrm{NaCl}$, $50 \mathrm{mM}$ Tris- $\mathrm{HCl}(\mathrm{pH} 7.5), 1 \% \mathrm{NP}-40,0.5 \%$ sodium deoxycholate, $0.1 \% \mathrm{SDS}$, $1 \mathrm{mM}$ EDTA and protease inhibitors. Whole-cell lysates were immunoprecipitated for $2 \mathrm{~h}$ using anti-FLAG M2 agarose beads (Sigma-Aldrich). Immune complexes were washed with lysis buffer and denaturated with $2 \times$ SDS sample buffer. Denatured proteins were detected by western blot analysis. For the immunoprecipitation of endogenous TAZ, an anti-TAZ antibody (BD Biosciences, \#560235) was used.

Chromatin immunoprecipitation. Chromatin immunoprecipitation was performed using a previously described method. ${ }^{48}$ The PCR primer sequences were as follows: osteocalcin promoter-F, 5'-CTGAACTGGGCAAATGAGGACA-3'; osteocalcin promoter-R, $5^{\prime}$-AGGGGATGCTGCCAGGACTAAT-3'; Runx2 promoter-F, 5'-CAGTGGTAGGCA GTCCCACTTTAC-3'; Runx2 promoter-R, 5'-GGCTG GTAGTGACCTGCAGAGAT-3'.

siRNA transfection. C3H10T1/2 cells were transfected with mPP1A siRNAs using Lipofectamine 2000 reagent (Invitrogen), and, $24 \mathrm{~h}$ later, the transfected cells were incubated in culture medium containing control or Wnt3a-conditioned media for $24 \mathrm{~h}$. The PP1A target sequences were as follows:

mPP1A target sequence \#1, 5' - AAGACGTTCACTGACTGCTTC-3';

mPP1A target sequence \#2, 5'-CCATTCTTCTGGAGCTTGA-3';

mPP1A target sequence \#3, 5' - CAAAGCAAGCAAAGAAATA-3'.

In vitro ubiquitination assay. For the ubiquitination assay, $3.6 \times 10^{6} 293 \mathrm{~T}$ cells were plated per $100 \mathrm{~mm}$ culture dish and transfected with HA-Ub and FLAGTAZ expression plasmids. Subsequently, the transfected 293T cells were treated with Wnt3a-conditioned medium for $12 \mathrm{~h}$. FLAG-TAZ-conjugated ubiquitin was purified from total cell lysates using anti-FLAG M2 agarose beads, and TAZ ubiquitination was analysed by immunoblotting.

Immunocytochemistry. C3H10T1/2 cells were seeded onto 24-well culture plates, and, $16 \mathrm{~h}$ later, the culture medium was replaced with $\mathrm{C} 3 \mathrm{H} 10 \mathrm{~T} 1 / 2$ cell growth media in the presence or absence of Wnt3a. After $24 \mathrm{~h}$, the cells were washed with ice-cold PBS, fixed with $4 \%$ paraformaldehyde for $15 \mathrm{~min}$, permeabilised with $0.05 \%$ Triton X-100 in PBS for 10 min and blocked with $3 \%$ BSA. Subsequently, the cells were incubated with TAZ-specific primary antibodies (prepared with the TAZ-specific peptide SSGGHPGPRLAGGA). After primary antibody staining, the cells were incubated with FITC-conjugated secondary antibodies for $90 \mathrm{~min}$. The fluorescence signal was analysed by microscopy.

Cytosolic and nuclear fractionation. Cells were incubated with or without Wnt3a-conditioned medium before collecting and lysis with fractionation buffer $(20 \mathrm{mM}$ HEPES, $10 \mathrm{mM} \mathrm{KCl}, 2 \mathrm{mM} \mathrm{MgCl}, 1 \mathrm{mM}$ EDTA and protease inhibitor) for $5 \mathrm{~min}$ at $4{ }^{\circ} \mathrm{C}$. The lysates were then passed through a $20 \mathrm{G}$ needle 20 times using a syringe and centrifuged at $10000 \times g$ for $10 \mathrm{~min}$ (supernatant: cytosolic fraction; pellet: nuclear fraction). The pellets were lysed with RIPA buffer for $20 \mathrm{~min}$ at $4{ }^{\circ} \mathrm{C}$ (supernatant: nuclear fraction). Cytosolic and nuclear fractions were analysed by western blot analysis.

\section{Conflict of Interest}

The authors declare no conflict of interest.

Acknowledgements. This work was supported by Basic Science Research Programs (2011-0022926) of the National Research Foundation of Korea (NRF) and grants (A120349 and A120476) from the Korea Healthcare Technology R\&D Project, Republic of Korea.

1. Kanai F, Marignani PA, Sarbassova D, Yagi R, Hall RA, Donowitz M et al. TAZ: a novel transcriptional co-activator regulated by interactions with 14-3-3 and PDZ domain proteins. Embo J 2000; 19: 6778-6791.

2. Hong JH, Hwang ES, McManus MT, Amsterdam A, Tian $Y$, Kalmukova $R$ et al. TAZ, a transcriptional modulator of mesenchymal stem cell differentiation. Science 2005; 309: 1074-1078.

3. Mahoney Jr WM, Hong JH, Yaffe MB, Farrance IK. The transcriptional co-activator TAZ interacts differentially with transcriptional enhancer factor-1 (TEF-1) family members. Biochem J 2005; 388(Pt 1): 217-225

4. Chan SW, Lim CJ, Loo LS, Chong YF, Huang C, Hong W. TEADs mediate nuclear retention of TAZ to promote oncogenic transformation. J Biol Chem 2009; 284: 14347-14358.

5. Zhang H, Liu CY, Zha ZY, Zhao B, Yao J, Zhao S et al. TEAD transcription factors mediate the function of TAZ in cell growth and epithelial-mesenchymal transition. J Biol Chem 2009; 284: $13355-13362$.

6. Park KS, Whitsett JA, Di Palma T, Hong JH, Yaffe MB, Zannini M. TAZ interacts with TTF-1 and regulates expression of surfactant protein-C. J Biol Chem 2004; 279: 17384-17390.

7. Murakami M, Nakagawa M, Olson EN, Nakagawa O. A WW domain protein TAZ is a critical coactivator for TBX5, a transcription factor implicated in Holt-Oram syndrome. Proc Natl Acad Sci USA 2005; 102: 18034-18039.

8. Murakami M, Tominaga J, Makita R, Uchijima $Y$, Kurihara $Y$, Nakagawa $O$ et al. Transcriptional activity of Pax3 is co-activated by TAZ. Biochem Biophys Res Commun 2006; 339: 533-539.

9. Varelas X, Sakuma R, Samavarchi-Tehrani P, Peerani R, Rao BM, Dembowy J et al. TAZ controls Smad nucleocytoplasmic shuttling and regulates human embryonic stem-cell self-renewal. Nat Cell Biol 2008; 10: 837-848.

10. Jeong H, Bae S, An SY, Byun MR, Hwang JH, Yaffe MB et al. TAZ as a novel enhancer of MyoD-mediated myogenic differentiation. Faseb J 2010; 24: 3310-3320.

11. Jang EJ, Jeong H, Han KH, Kwon HM, Hong JH, Hwang ES. TAZ Suppresses NFAT5 Activity through Tyrosine Phosphorylation. Mol Cell Biol 2012; 32: 4925-4932.

12. Zhao B, Tumaneng K, Guan KL. The Hippo pathway in organ size control, tissue regeneration and stem cell self-renewal. Nat Cell Biol 2011; 13: 877-883.

13. Varelas $X$, Wrana JL. Coordinating developmental signaling: novel roles for the Hippo pathway. Trends Cell Biol 2012; 22: 88-96.

14. Pan D. The hippo signaling pathway in development and cancer. Dev Cell 2010; 19: 491-505.

15. Ramos A, Camargo FD. The Hippo signaling pathway and stem cell biology. Trends Cell Biol 2012; 22: 339-346.

16. Angers S, Moon RT. Proximal events in Wnt signal transduction. Nat Rev Mol Cell Biol 2009; 10: 468-477.

17. Clevers $H$, Nusse R. Wnt/beta-catenin signaling and disease. Cell 2012; 149: 1192-1205.

18. MacDonald BT, Tamai $\mathrm{K}, \mathrm{He} \mathrm{X}$. Wnt/beta-catenin signaling: components, mechanisms, and diseases. Dev Cell 2009; 17: 9-26.

19. He X, Semenov M, Tamai K, Zeng X. LDL receptor-related proteins 5 and 6 in Wnt/beta-catenin signaling: arrows point the way. Development 2004; 131: 1663-1677.

20. Ferrari SL, Deutsch S, Choudhury U, Chevalley T, Bonjour JP, Dermitzakis ET et al. Polymorphisms in the low-density lipoprotein receptor-related protein 5 (LRP5) gene are 
associated with variation in vertebral bone mass, vertebral bone size, and stature in whites. Am J Hum Genet 2004; 74: 866-875.

21. Kwee ML, Balemans W, Cleiren E, Gille JJ, Van Der Blij F, Sepers JM et al. An autosoma dominant high bone mass phenotype in association with craniosynostosis in an extended family is caused by an LRP5 missense mutation. J Bone Miner Res 2005; 20: 1254-1260.

22. Balemans W, Devogelaer JP, Cleiren E, Piters E, Caussin E, Van Hul W. Novel LRP5 missense mutation in a patient with a high bone mass phenotype results in decreased DKK1-mediated inhibition of Wnt signaling. J Bone Miner Res 2007; 22: 708-716.

23. Kato M, Patel MS, Levasseur R, Lobov I, Chang BH, Glass DA 2nd et al. Cbfa1-independent decrease in osteoblast proliferation, osteopenia, and persistent embryonic eye vascularization in mice deficient in Lrp5, a Wnt coreceptor. J Cell Biol 2002; 157: 303-314.

24. Bennett CN, Ouyang H, Ma YL, Zeng Q, Gerin I, Sousa KM et al. Wnt10b increases postnatal bone formation by enhancing osteoblast differentiation. J Bone Miner Res 2007; 22: 1924-1932.

25. Bennett CN, Longo KA, Wright WS, Suva LJ, Lane TF, Hankenson KD et al. Regulation of osteoblastogenesis and bone mass by Wnt10b. Proc Natl Acad Sci USA 2005; 102: 3324-3329.

26. Glass 2nd DA, Bialek P, Ahn JD, Starbuck M, Patel MS, Clevers $\mathrm{H}$ et al. Canonical Wnt signaling in differentiated osteoblasts controls osteoclast differentiation. Dev Cell 2005; 8: 751-764.

27. Holmen SL, Zylstra CR, Mukherjee A, Sigler RE, Faugere MC, Bouxsein ML et al. Essential role of beta-catenin in postnatal bone acquisition. J Biol Chem 2005; 280: 21162-21168.

28. Kramer I, Halleux C, Keller H, Pegurri M, Gooi JH, Weber PB et al. Osteocyte Wnt/betacatenin signaling is required for normal bone homeostasis. Mol Cell Biol 2010; 30 3071-3085.

29. Azzolin L, Zanconato F, Bresolin S, Forcato M, Basso G, Bicciato S et al. Role of TAZ as Mediator of Wnt Signaling. Cell 2012; 151: 1443-1456.

30. Tian Y, Kolb R, Hong JH, Carroll J, Li D, You J et al. TAZ promotes PC2 degradation through a SCFbeta-Trcp E3 ligase complex. Mol Cell Biol 2007; 27: 6383-6395.

31. Liu CY, Zha ZY, Zhou X, Zhang H, Huang W, Zhao D et al. The hippo tumor pathway promotes TAZ degradation by phosphorylating a phosphodegron and recruiting the SCF\{beta\}-TrCP E3 ligase. J Biol Chem 2010; 285: 37159-37169.

32. Huang W, Lv X, Liu C, Zha Z, Zhang H, Jiang $Y$ et al. The N-terminal phosphodegron targets TAZ/WWTR1 protein for SCFbeta-TrCP-dependent degradation in response to phosphatidylinositol 3-kinase inhibition. J Biol Chem 2012; 287: 26245-26253.

33. Lei QY, Zhang H, Zhao B, Zha ZY, Bai F, Pei XH et al. TAZ promotes cell proliferation and epithelial-mesenchymal transition and is inhibited by the hippo pathway. Mol Cell Biol 2008; 28: 2426-2436.
34. Liu CY, Lv X, Li T, Xu Y, Zhou X, Zhao S et al. PP1 cooperates with ASPP2 to dephosphorylate and activate TAZ. J Biol Chem 2011; 286: 5558-5566.

35. Varelas X, Miller BW, Sopko R, Song S, Gregorieff A, Fellouse FA et al. The Hippo pathway regulates Wnt/beta-catenin signaling. Dev Cell 2010; 18: 579-591.

36. Imajo M, Miyatake K, limura A, Miyamoto A, Nishida E. A molecular mechanism that links Hippo signalling to the inhibition of Wnt/beta-catenin signalling. Embo J 2012; 31: $1109-1122$.

37. Heallen T, Zhang M, Wang J, Bonilla-Claudio M, Klysik E, Johnson RL et al. Hippo pathway inhibits Wnt signaling to restrain cardiomyocyte proliferation and heart size. Science 2011; 332: 458-461.

38. Xin M, Kim Y, Sutherland LB, Qi X, McAnally J, Schwartz RJ et al. Regulation of insulin-like growth factor signaling by Yap governs cardiomyocyte proliferation and embryonic heart size. Sci Signal 2011; 4: ra70.

39. Konsavage Jr WM, Kyler SL, Rennoll SA, Jin G, Yochum GS. Wnt/beta-catenin signaling regulates Yes-associated protein (YAP) gene expression in colorectal carcinoma cells. J Biol Chem 2012; 287: 11730-11739.

40. Tian Y, Kolb R, Hong JH, Carroll J, Li D, You J et al. TAZ promotes PC2 degradation through a SCFbeta-Trcp E3 ligase complex. Mol Cell Biol 2007; 27: 6383-6395.

41. Makita R, Uchijima Y, Nishiyama K, Amano T, Chen Q, Takeuchi T et al. Multiple renal cysts, urinary concentration defects, and pulmonary emphysematous changes in mice lacking TAZ. Am J Physiol Renal Physiol 2008; 294: F542-F553.

42. Zaidi SK, Sullivan AJ, Medina R, Ito $Y$, van Wijnen AJ, Stein JL et al. Tyrosine phosphorylation controls Runx2-mediated subnuclear targeting of YAP to repress transcription. Embo J 2004; 23: 790-799.

43. Clement-Lacroix P, Ai M, Morvan F, Roman-Roman S, Vayssiere B, Belleville C et al. Lrp5-independent activation of Wnt signaling by lithium chloride increases bone formation and bone mass in mice. Proc Natl Acad Sci USA 2005; 102: 17406-17411.

44. Cui Y, Niziolek PJ, MacDonald BT, Zylstra CR, Alenina N, Robinson DR et al. Lrp5 functions in bone to regulate bone mass. Nat Med 2011; 17: 684-691.

45. Babij P, Zhao W, Small C, Kharode Y, Yaworsky PJ, Bouxsein ML et al. High bone mass in mice expressing a mutant LRP5 gene. J Bone Miner Res 2003; 18: 960-974.

46. Hossain Z, Ali SM, Ko HL, Xu J, Ng CP, Guo K et al. Glomerulocystic kidney disease in mice with a targeted inactivation of Wwtr1. Proc Natl Acad Sci USA 2007; 104: 1631-1636.

47. Yang JY, Cho SW, An JH, Jung JY, Kim SW, Kim SY et al. Osteoblast-targeted overexpression of TAZ increases bone mass in vivo. PLoS One 2013; 8: e56585.

48. Byun MR, Jeong H, Bae SJ, Kim AR, Hwang ES, Hong JH. TAZ is required for the osteogenic and anti-adipogenic activities of kaempferol. Bone 2012; 50: 364-372.

Supplementary Information accompanies this paper on Cell Death and Differentiation website (http://www.nature.com/cdd) 